\title{
Laparoscopic Assisted Percutaneous Microwave Ablation for Hepatocellular Carcinoma Close to Large Hepatic Vessels
}

\author{
Mohamed Mogahed ${ }^{1, ~ *, ~ B a h a a ~ E l ~ W a k e e l ~}{ }^{1}$, Ashraf El Kholy ${ }^{1}$, Wessam Moustafa Abdellatif ${ }^{2}$, \\ Ashraf Anas Zytoon ${ }^{3}$, Mohamed Manaa ${ }^{4}$, Nashwa Said Ghanem ${ }^{5}$ \\ ${ }^{1}$ Surgical Department, National Hepatology \& Tropical Medicine Research Institute "NHTMRI”, Cairo, Egypt \\ ${ }^{2}$ Radiology Department, National Hepatology \& Tropical Medicine Research Institute "NHTMRI", Cairo, Egypt \\ ${ }^{3}$ Radiodiagnosis Department, Faculty of Medicine, Menoufia University, Menoufia, Egypt \\ ${ }^{4}$ Tropical Medicine Department, National Hepatology \& Tropical Medicine Research Institute "NHTMRI”, Cairo, Egypt \\ ${ }^{5}$ Internal Medicine Department, Faculty of Medicine, Cairo University, Cairo, Egypt \\ Email address: \\ Mogahed1968@yahoo.com (M. Mogahed) \\ ${ }^{*}$ Corresponding author
}

\section{To cite this article:}

Mohamed Mogahed, Bahaa ElWakeel, Ashraf ElKholy, Wessam Moustafa Abdellatif, Ashraf Anas Zytoon, Mohamed Manaa, Nashwa Said Ghanem. Laparoscopic Assisted Percutaneous Microwave Ablation for Hepatocellular Carcinoma Close to Large Hepatic Vessels. Journal of Surgery. Vol. 7, No. 5, 2019, pp. 132-137. doi: 10.11648/j.js.20190705.14

Received: May 13, 2019; Accepted: June 25, 2019; Published: August 29, 2019

\begin{abstract}
Background: Hepatocellular carcinoma (HCC) is one of the most common cancers worldwide. It was believed that ablation for tumors close to large vessels should be avoided for the concern of major complications. Microwave ablation (MWA) seemed to be a better choice than radiofrequency ablation (RFA) in treating HCC close to large vessels ( $\geq 3 \mathrm{~mm}$ ), for its advantages of a lower susceptibility to heat-sink effects (blood-vessel-mediated cooling), as well as the ability to achieve larger tumor volumes in shorter time. The aim of this study is to evaluate safety and efficacy of MWA for HCC close to large vessels. Methods: 32 patients with $52 \mathrm{HCC}$ lesions were included, all patients have a lesion close to large vessel. 14 patients had single lesion, 16 had two lesions and 2 had three lesions. All patients are Child-Pugh A or B within Milan Criteria and class A disease Barcelona Clinic Liver Cancer (BCLC). Results: This study was conducted on 32 HCC patients 24 males and 8 females with median age 63 years. $32 \mathrm{HCC}$ lesions were close to large vessel while 20 lesions were not close to large vessel. Laparoscopic assisted percutaneous MWA was decided as the treatment of choice for all patients. two patients (6\%) had major complications (one patient had right portal vein thrombosis and the other patient had intra-hepatic hematoma), 3 patients (9\%) had minor complications in the form of skin burn. Local tumor progression (LTP) occurred in 2 lesions (3.8\% of lesions). Conclusion: laparoscopic assisted percutaneous MWA proved to be a safe and effective as a management for HCC close to large vessels.
\end{abstract}

Keywords: Laparoscopic Assisted Percutaneous, Microwave Ablation, Hepatocellular Carcinoma

\section{Introduction}

Hepatocellular carcinoma (HCC) is one of the most common cancers worldwide. [1] Egypt has a high incidence of HCC (around $21 \%$ of cirrhotic Egyptian patients). This may be attributed to high prevalence of HCV in Egypt. [2] $\mathrm{HCC}$ has poor prognosis due to high degree of malignancy, high recurrence rates and the impaired liver function associated with the disease. Surgical resection is the optimal treatment for early $\mathrm{HCC}$ in patients with compensated cirrhosis but the majority of HCC patients are not candidates for surgical resection because of their poor hepatic functional reserve, advanced tumors, and because a tumor location close to major intrahepatic vessels and multi-focal tumors preclude a negative margin resection. [3] Thermal ablation therapies have been widely used for the local treatment of HCC. [4] It 
was believed that ablation for tumors close to large vessels should be avoided for the concern of major complications. [5, 6] Microwave ablation is electromagnetic wave which is better than radiofrequency ablation (RFA) in treating HCC close to large vessels, for its advantages of a lower susceptibility to heat-sink effects (blood-vessel-mediated cooling) and its ability to achieve larger tumor volumes in shorter time. [7]

The aim of this study is to evaluate safety and efficacy of MWA of HCC close to large vessels.

\section{Patients and Methods}

This prospective study conducted in National Hepatology and Tropical Medicine Research Institute (NHTMRI), Cairo, Egypt from February 2014 to December 2018 on 32 patients (24 males and 8 females) with 52 HCC lesions. The median age of the patients was 63 years. All patients had lesion close to large vessel $\geq 3 \mathrm{~mm}$ diameter (hepatic vein, portal vein, inferior vena cava). $32 \mathrm{HCC}$ lesions were close to large vessel while 20 lesions were not close to large vessel. 14 patients had single lesion, 16 had two lesions and 2 patients had three lesions as shown in (figure 1). The median tumor diameter was $2.4 \mathrm{~cm}, 45$ lesions were $\leq 3 \mathrm{~cm}$ and 7 lesions were $>3 \mathrm{~cm}$ these 7 lesions were close to large vessel. All patients had cirrhosis due to hepatitis $\mathrm{C}$ or B virus infection. They were within Milan Criteria and they were class A disease Barcelona Clinic Liver Cancer (BCLC). The diagnosis of HCC was based on American Association for the Study of Liver Diseases (AASLD) radiological criteria or histology. [8, 9] Four lesions of the 52 lesions were discovered intraoperative by intraoperative laparoscopic ultrasound. The nature of the procedure was explained and informed consents were taken from all participating patients. Description of patients and HCC lesions included in this study is summarized in (table 1).

Table 1. Description of patients and HCC lesions included in the study.

\begin{tabular}{ll}
\hline Number of patients & 32 \\
Median Age (years) & 63 \\
Male/female & $24 / 8$ patients \\
Within Milan criteria n (\%) & $32(100 \%)$ \\
BCLC Stage A n (\%) & $32(100 \%)$ \\
Liver cirrhosis n (\%) & $32(100 \%)$ \\
Child - Pugh n (\%) & \\
Class A & $25(78 \%)$ \\
Class B & $7(22 \%)$ \\
Class C & $0(0 \%)$ \\
Total number of HCC lesions n (\%) & $52(100 \%)$ \\
HCC Close to large vessel n $(\%)$ & $32(62 \%)$ \\
HCC Not close to large vessel n $(\%)$ & $20(38 \%)$ \\
HCC lesions $\leq 3 \mathrm{~cm}$ n $(\%)$ & $45(87 \%)$ \\
HCC lesions $>3 \mathrm{~cm}$ and $<5 \mathrm{~cm} \mathrm{n}(\%)$ & $7(13 \%)$ \\
\hline
\end{tabular}

\subsection{Surgical Procedure}

Procedures were performed under general anesthesia with the patient in a supine position. Pneumoperitoneum was induced and inflation pressure was maintained at $10-13 \mathrm{mmHg}$. A second trocar was inserted in the right or left upper quadrant according to the site of liver lesions to allow the passage of the ultrasound probe. After the peritoneal cavity had been explored, laparoscopic intraoperative ultrasound (LIOUS) was performed to complete the disease staging, confirm the tumor location and establish its relationship with the major hepatic vasculature. MW ablation needles were inserted percutaneous and placed inside the lesion under laparoscopic intraoperative ultrasound (LIOUS) guidance as shown in (figure 2). Tumor ablation was confirmed in all patients during the procedure by LIOUS then a drain was inserted to be removed postoperatively. The mean operative time was 88 minutes and the median MW ablation time for each HCC lesion was 6 minutes (range 4-12 $\mathrm{min}$ ).

\subsection{Microwave Ablation (MWA)}

Is electromagnetic energy, not electric current, no need for grounding pads. The devices consist of three basic parts: generator, flexible cable and antenna. In the absence of current flow, the electromagnetic field creates a rapid and homogeneous heating of tissue and subsequently coagulation necrosis. The best heating effect is achieved in tissues with a high content of water and the worst is observed in fat. [10] Another mechanism of MWA function is ionic polarization with conversion of kinetic energy into heat. A more homogeneous, larger ablation zone that is easily predicted is feasible and the heat-sink effect is attenuated. [11, 12] The faster heating and higher temperatures provided by microwave energy may be a reason for the reduced heat-sink effect. [13]

Microwave system used in our institution in this study is (AMICA- GEN Apparatus for microwave ablation system) with frequency of $2,450 \mathrm{MHz}$ and generators capable of producing 140 Watts of power. AMICA ${ }^{\mathrm{TM}}$ Probe has Sharp trocar point for easy penetration into tissues. Very good US visibility. Internal water cooling to avoid shaft overheating. Built-in thermocouple for probe temperature monitoring. Embedded memory chip for probe identification by AMICAGEN, providing enhanced ease and safety of use. This MWA system provides large and fast ablations: more than $4 \mathrm{~cm}$ in diameter in 10 minutes, with a single probe.

\subsection{Postoperative Follow-up}

The median hospital stay was 3 days. Abdomino-pelvic ultrasound was done for all patients before discharge from hospital. The local efficacy of ablation and local tumor progression was evaluated with tri-phasic CT and/or MRI and serum alpha fetoprotein in all patients after one month, every 3 months for the first year and every 6 months thereafter. Follow up period was at least 12 months. Local tumor progression (LTP) is defined as enhancement at the arterial phase with washout lesion at the delayed phase of the contrast-enhanced imaging examination (tri-phasic computed tomography and magnetic resonance imaging) inside or abutting the ablation zone during follow-up.

\subsection{Statistical Analysis}

Descriptive statistics were reported as frequencies and percents. 


\section{Results}

In this study 32 patients with 52 HCC lesions underwent laparoscopic assisted percutaneous MWA. There were 14 patients $(44 \%)$ had single lesion, $16(50 \%)$ had two lesions and 2 patients $(6 \%)$ had three lesions. The median tumor diameter was $2.4 \mathrm{~cm}, 45$ lesions $(87 \%)$ were $\leq 3 \mathrm{~cm}$ and 7 lesions $(13 \%)$ were $>3 \mathrm{~cm}$ and $<5 \mathrm{~cm} .32 \mathrm{HCC}$ lesions $(62 \%)$ were close to large vessel while 20 lesions (38\%) were not close to large vessel as shown in (figure 3 ). The followup period was at least 12 months.

The median postoperative hospital stay was 3 days. There was no perioperative mortality. Technical success was $100 \%$, no conversion to open surgery, all lesions were completely ablated as confirmed by LIOUS. According to SIR classification [14] system for complications by outcome two patients $(6 \%)$ had major complications (one patient had right portal vein thrombosis and the other patient had intra-hepatic hematoma), these lesions were $>3 \mathrm{~cm}$ and close to large vessels. Patients with complications were treated conservatively with medical treatment and no surgical intervention was required. Minor complications occurred in 3 patients $(9 \%)$ in the form of skin burn. Follow up period was at least 12 months with tri-phasic CT and/or MRI and serum alpha fetoprotein for all patients after one month, every 3 months for the first year and every 6 months thereafter (figure 4 show triphasic MRI before and after MWA). Local tumor progression (LTP) occurred in 2 lesions $(3.8 \%$ of lesions) that were $>3 \mathrm{~cm}$ at 12 months follow up visit.

\section{Number of patiens with single, 2 and 3 HCC lesions}

m single lesion in14 patients $\quad 2$ lesions in 16 patients $\quad 3$ lesions in 2 patients

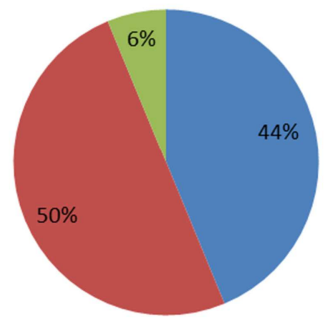

Figure 1. Number of patients with single, 2 and 3 HCC lesions.

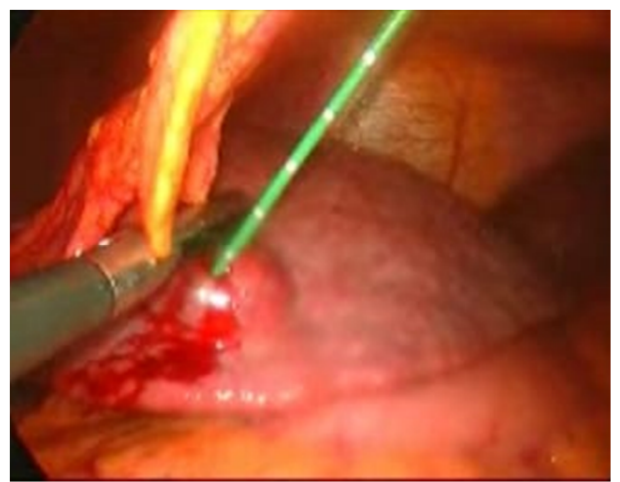

Figure 2. Laparoscopic Assisted Percutaneous MW ablation guided by (LIOUS).

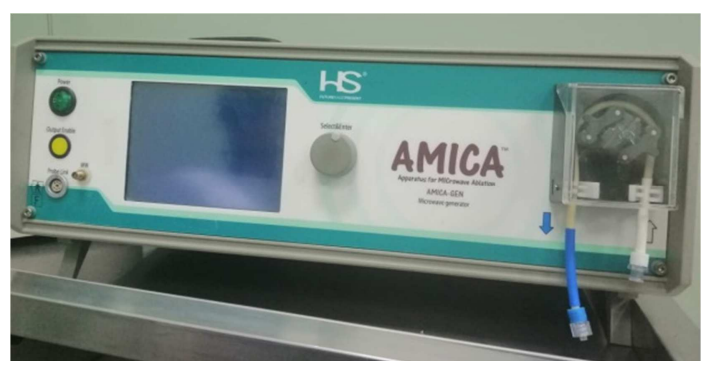

Figure 3. Microwave Ablation Device.

\section{Relation of HCC to large vessels}

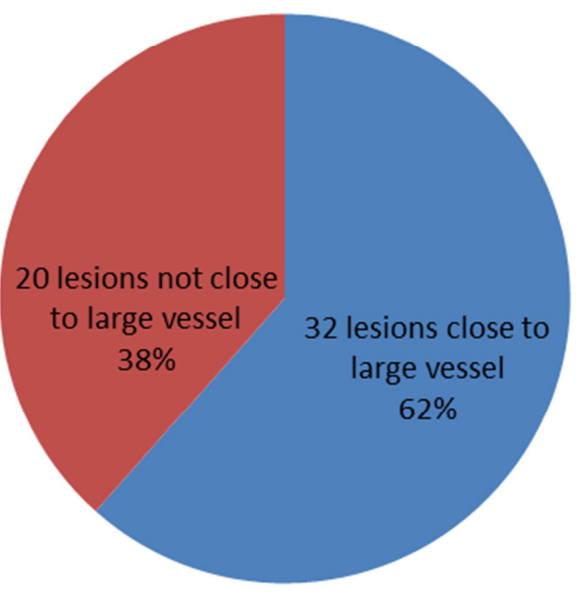

Figure 4. Relation of HCC to large vessels.

\section{Discussion}

Thermal ablation currently represents the first-line treatment option for early stage unresectable HCC. Unresectable HCC includes single lesion in a deep location, multinodular disease in patients with deteriorated liver function. These procedures are usually performed by percutaneous approach but in particular conditions as in cases of nodules in "at-risk" location laparoscopic ablation is recommended. [15]

Microwave ablation is electromagnetic wave which is better than radiofrequency ablation (RFA) in treating HCC close to large vessels, for its advantages of a lower susceptibility to heat-sink effects and its ability to achieve larger tumor volumes in shorter time. [7] In MWA multiple antennae can be simultaneously activated without the electrical interference phenomena observed in RFA, thus allowing more rapid treatment of large or multifocal tumors. [16]

The aim of this study is to evaluate safety and efficacy of MWA of HCC close to large vessels.

In the current study we present single-center experience of laparoscopic assisted percutaneous MWA for HCC. There was no perioperative mortality. Technical success was $100 \%$, no conversion to open surgery, all lesions were completely ablated these results are comparable to Simo et al 2011 but superior to results of 3 retrospective percutaneous 
sonographically guided studies that reported complete ablation rates of $89 \%-94 \%$, our results may be explained by the use of laparoscopic technique guided by LIOUS for complete ablation confirmation. [17-20]

As regard safety in the current study there was no perioperative mortality, 2 patients had major complications $(6 \%)$ (one patient had right portal vein thrombosis and the other patient had intra-hepatic hematoma) patients were treated conservatively with medical treatment and no surgical intervention was required. These results are comparable to the results of a large multicenter Italian study in which the major complication rate was $2.9 \%$ and the peri-procedural mortality rate $<0.01 \%$ after MWA for HCC. [21]
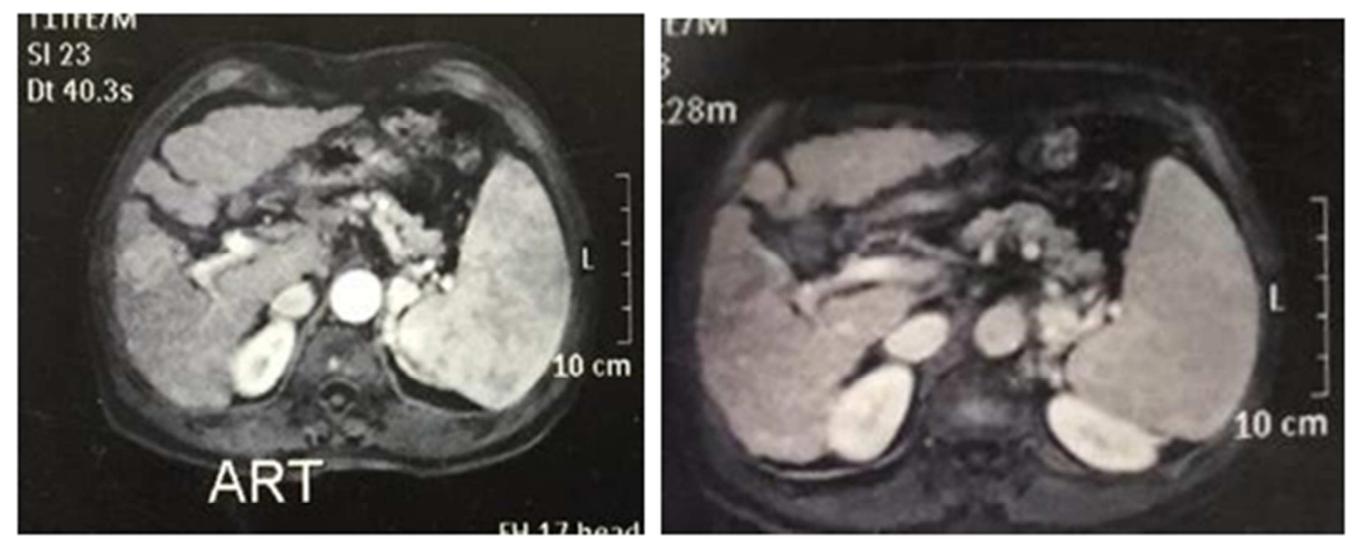

la

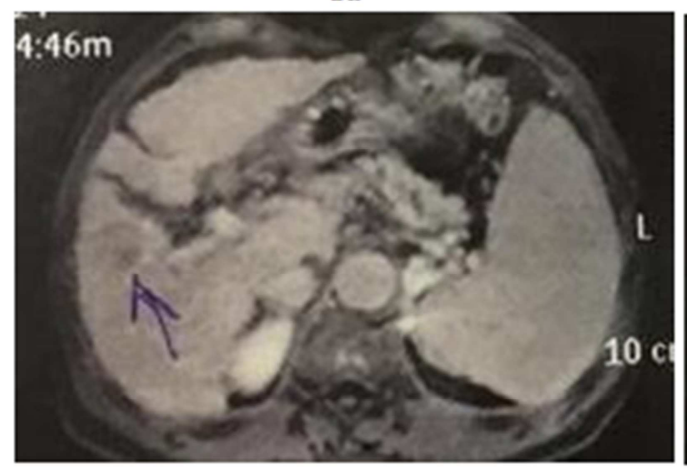

lb

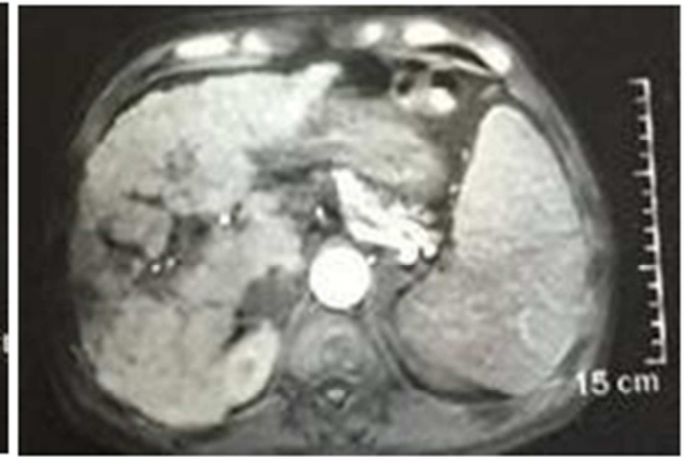

lc

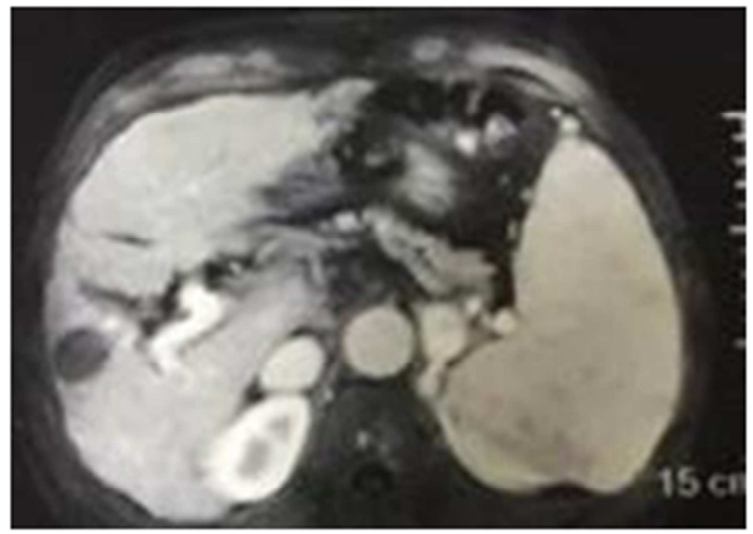

2b 2a

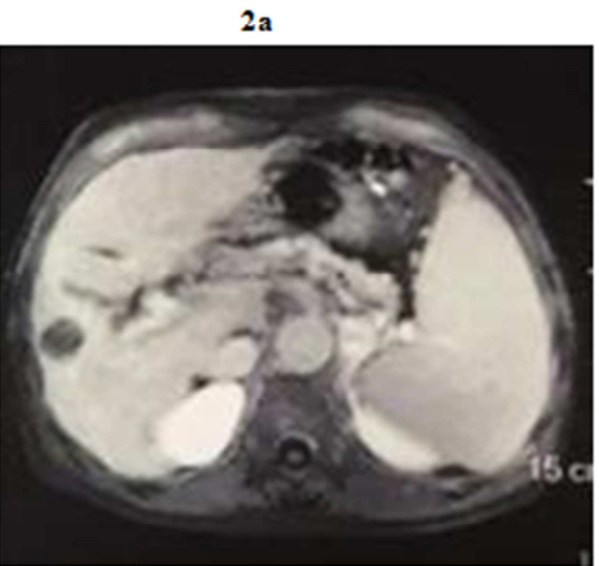

$2 \mathrm{c}$

Figure 5. Show triphasic MRI before and after MWA.

1a: Dynamic MRI before ablation (arterial phase): well defined right lobe segment V HCC with homogenous arterial enhancement - 5mm from right portal vein branch.

1b: Dynamic MRI before ablation (portal phase): HCC showing early wash out.

1c: Dynamic MRI before ablation (venous phase): HCC shows characteristic wash out.

2a: Dynamic MRI after ablation (arterial phase): right lobe segment V non enhanced area denoting good ablation with no definite tumoral reactivity.

2b: Dynamic MRI after ablation (portal phase): the abated area still shows no enhancement with patent homogeneously enhanced right portal vein branch.

2c: Dynamic MRI after ablation (venous phase): still no enhancement at the ablation area denoting good ablation. 
Portal veins thrombosis occurs more frequently than hepatic veins and should be treated cautiously during MWA, particularly in patients with liver cirrhosis. [22] The mechanism behind the increased rate of portal veins thrombosis compared with hepatic veins is related to differences in flow velocity, flow pattern, and total blood flow. Portal veins have slower blood flow because of drainage into high-resistance hepatic sinusoids. This relatively sluggish flow is exacerbated in patients with cirrhosis and portal hypertension, who have even higher sinus pressures and slower ante grade portal vein flow. [23] Slow flow is less effective at dissipating heat, resulting in vessel occlusion. [24]

In the current study local tumor progression (LTP) occurred in 2 lesions $(2 / 52,3.8 \%$ of lesions) that were $>3 \mathrm{~cm}$ and close to large vessels at 12 months follow up visit. These results are comparable to Abdelaziz et al. with rate of $3.9 \%$ but superior to Chiang J, et al. 2016, Vogl et al and Zhang et al with recurrence rate of $9.6 \%, 8.3 \%$ and $10,5 \%$ respectively. $[22,25-27]$ All these last-mentioned studies reported lower recurrence rates when MWA was compared to RFA.

\section{Conclusion}

Laparoscopic assisted percutaneous MWA is safe and effective management for HCC close to large vessels. Combination of laparoscopic intraoperative ultrasound LIOUS with Laparoscopic assisted percutaneous MWA helps in accurate detection of tumor location, its relation to large vessels, helpful in confirmation of tumor ablation and can detect new HCC lesions not detected preoperatively with lower recurrence rates.

\section{References}

[1] Himoto T, Kurokohchi K, Watanabe S, Masaki T. Recent advances in radiofrequency ablation for the management of hepatocellular carcinoma. Hepat Mon. 2012; 12: e5945. [PMC free article] [PubMed].

[2] Atti EA (2015) HCC Burden in Egypt. Gastroentero J Hepatol Open Access 2 (3): 00045.

[3] Fong Y, Sun RL, Jarnagin W, Blumgart LH. An analysis of 412 cases of hepatocellular carcinoma at a Western centre. Ann Surg. 1999; 229: 790-799.; discussion 799-800.

[4] Sakaguchi H, Seki S, Tsuji K, Teramoto K, Suzuki M, Kioka $\mathrm{K}$, et al. Endoscopic thermal ablation therapies for hepatocellular carcinoma: a multi-centre study. Hepatol Res. 2009; 39: 47-52. [PubMed].

[5] Parkin DM, Bray F, Ferlay J, Pisani P. Global cancer statistics, 2002. CA Cancer J Clin. 2005; 55: 74-108. [PubMed].

[6] Yang JD, Roberts LR. Epidemiology and management of hepatocellular carcinoma. Infect Dis Clin North Am. 2010; 24: 899-919, viii. [PMC free article] [PubMed].

[7] Forner A, Reig ME, de Lope CR, Bruix J. Current strategy for staging and treatment: the BCLC update and future prospects.
Semin Liver Dis. 2010; 30: 61-74. [PubMed].

[8] Bruix J, Sherman M. Management of hepatocellular carcinoma. Hepatology. 2005; 42: 1208-1236. [PubMed].

[9] Bruix J, Sherman M. Management of hepatocellular carcinoma: an update. Hepatology. 2011; 53: 1020-1022.

[10] Brace CL. Radiofrequency and microwave ablation of the liver, lung, kidney, and bone: what are the differences? Curr Probl Diagn Radiol. 2009; 38: 135-143. [PMC free article] [PubMed].

[11] Simon CJ, Dupuy DE, Mayo-Smith WW. Microwave ablation: principles and applications. Radiographics. 2005; 25 Suppl 1: S69-S83. [PubMed].

[12] Yu NC, Raman SS, Kim YJ, Lassman C, Chang X, Lu DS. Microwave liver ablation: influence of hepatic vein size on heat-sink effect in a porcine model. J Vasc Interv Radiol. 2008; 19: 1087-1092. [PubMed].

[13] Lloyd DM, Lau KN, Welsh F, Lee KF, Sherlock DJ, Choti MA, Martinie JB, Iannitti DA. International multicentre prospective study on microwave ablation of liver tumours: preliminary results. HPB (Oxford) 2011; 13: 579-585.

[14] Ahmed M, Solbiati L, Brace CL, Breen DJ, Callstrom MR, Charboneau JW, Chen MH, Choi BI, de Baère T, Dodd GD 3rd, Dupuy DE, Gervais DA, Gianfelice D, et al. Imageguided tumor ablation: standardization of terminology and reporting criteria-a 10-year update. Radiology. 2014; 273: 241-60.

[15] Facciorusso A, Serviddio G, Muscatiello N. Local ablative treatments for hepatocellular carcinoma: An updated review. World J Gastrointest Pharmacol Ther. 2016 Nov 6; 7 (4): 477 489. Review.

[16] McWilliams JP, Yamamoto S, Raman SS, Loh CT, Lee EW, Liu DM, Kee ST. Percutaneous ablation of hepatocellular carcinoma: current status. J Vasc Interv Radiol. 2010; 21: S204-S213.

[17] Simo KA, Sereika SE, Newton KN, Gerber DA. Laparoscopic-assisted microwave ablation for hepatocellular carcinoma: safety and efficacy in comparison with radiofrequency ablation. J Surg Oncol. 2011 Dec; 104 (7): 822-9. doi: 10.1002/jso.21933. Epub 2011 Apr 25. PMID: 21520094.

[18] Dong B, Liang P, Yu X, Su L, Yu D, Cheng Z, Zhang J. Percutaneous sonographically guided microwave coagulation therapy for hepatocellular carcinoma: results in 234 patients. AJR Am J Roentgenol. 2003; 180: 1547-1555. [PubMed].

[19] Liang P, Dong B, Yu X, Yu D, Wang Y, Feng L, Xiao Q. Prognostic factors for survival in patients with hepatocellular carcinoma after percutaneous microwave ablation. Radiology. 2005; 235: 299-307. [PubMed].

[20] Lu MD, Chen JW, Xie XY, Liu L, Huang XQ, Liang LJ, Huang JF. Hepatocellular carcinoma: US-guided percutaneous microwave coagulation therapy. Radiology. 2001; 221: 167172. [PubMed].

[21] Livraghi T, Meloni F, Solbiati L, Zanus G; Collaborative Italian Group using AMICA system. Complications of microwave ablation for liver tumors: results of a multicenter study. Cardiovasc Intervent Radiol. 2012; 35: 868-874. [PubMed]. 
[22] Chiang J, Cristescu M, Lee MH, Moreland A, Hinshaw JL, Lee FT, Brace CL. Effects of Microwave Ablation on Arterial and Venous Vasculature after Treatment of Hepatocellular Carcinoma. Radiology. 2016 Nov; 281 (2): 617-624. Epub 2016 Jun 3. PMID: 27257951.

[23] Perisic M, Ilic-Mostic T, Stojkovic M, Culafic D, Sarenac R. Doppler hemodynamic study in portal hypertension and hepatic encephalopathy. Hepatogastroenterology 2005; 52 (61): 156-160.

[24] Chiang J, Hynes K, Brace CL. Flow-dependent vascular heat transfer during microwave thermal ablation. Conf Proc IEEE Eng Med Biol Soc 2012; 2012: 5582-5585.

[25] Abdelaziz A, Elbaz T, Shousha HI, Mahmoud S, Ibrahim M,
Abdelmaksoud A, Nabeel M. Efficacy and survival analysis of percutaneous radiofrequency versus microwave ablation for hepatocellular carcinoma: an Egyptian multidisciplinary clinic experience. Surg Endosc. 2014; 28: 3429-3434. [PubMed].

[26] Vogl TJ, Farshid P, Naguib NN, Zangos S, Bodelle B, Paul J, Mbalisike EC, Beeres M, Nour-Eldin NE. Ablation therapy of hepatocellular carcinoma: a comparative study between radiofrequency and microwave ablation. Abdom Imaging. 2015; 40: 1829-1837. [PubMed].

[27] Zhang L, Wang N, Shen Q, Cheng W, Qian GJ. Therapeutic efficacy of percutaneous radiofrequency ablation versus microwave ablation for hepatocellular carcinoma. PLoS One. 2013; 8: e76119. [PMC free article] [PubMed]. 